OPEN ACCESS

Edited by:

Nehal Thakor

University of Lethbridge, Canada

Reviewed by:

Elif Pala,

Sanko University, Turkey

Piyush Khandelia,

Birla Institute of Technology and

Science, India

${ }^{*}$ Correspondence:

Sue-Ann Mok

sueann@ualberta.ca

Specialty section

This article was submitted to

RNA,

a section of the journal

Frontiers in Genetics

Received: 01 April 2021

Accepted: 19 May 2021

Published: 21 June 2021

Citation:

Budrass L, Fahlman RP and Mok SA (2021) Deciphering Network Crosstalk:

The Current Status and Potential of miRNA Regulatory Networks on the

HSP40 Molecular Chaperone

Network. Front. Genet. 12:689922.

doi: 10.3389/fgene.2021.689922

\section{Deciphering Network Crosstalk: The Current Status and Potential of miRNA Regulatory Networks on the HSP40 Molecular Chaperone Network}

\author{
Lion Budrass ${ }^{1}$, Richard P. Fahlman ${ }^{2,3}$ and Sue-Ann Mok ${ }^{1,2 *}$ \\ ${ }^{1}$ Neuroscience and Mental Health Institute, University of Alberta, Edmonton, AB, Canada, ${ }^{2}$ Department of Biochemistry, \\ University of Alberta, Edmonton, $A B$, Canada, ${ }^{3}$ Department of Oncology, University of Alberta, Edmonton, $A B$, Canada
}

Molecular chaperone networks fulfill complex roles in protein homeostasis and are essential for maintaining cell health. Hsp40s (commonly referred to as J-proteins) have critical roles in development and are associated with a variety of human diseases, yet little is known regarding the $\mathrm{J}$-proteins with respect to the post-transcriptional mechanisms that regulate their expression. With relatively small alterations in their abundance and stoichiometry altering their activity, post-transcriptional regulation potentially has significant impact on the functions of J-proteins. MicroRNAs (miRNAs) are a large group of non-coding RNAs that form a complex regulatory network impacting gene expression. Here we review and investigate the current knowledge and potential intersection of miRNA regulatory networks with the J-Protein chaperone network. Analysis of datasets from the current version of TargetScan revealed a great number of predicted microRNAs targeting J-proteins compared to the limited reports of interactions to date. There are likely unstudied regulatory interactions that influence chaperone biology contained within our analysis. We go on to present some criteria for prioritizing candidate interactions including potential cooperative targeting of J-Proteins by multiple miRNAs. In summary, we offer a view on the scope of regulation of J-Proteins through miRNAs with the aim of guiding future investigations by identifying key regulatory nodes within these two complex cellular networks.

Keywords: J-proteins, Hsp40, microRNAs, chaperone, target prediction

\section{INTRODUCTION}

MicroRNA (miRNA) networks of gene regulation and molecular chaperone networks both consist of complex webs of interactions with broad implications in shaping the proteome. In both networks, the activity of individual molecules impacts many target or client molecules, resulting in broad regulation of protein homeostasis (proteostasis) (Hipp et al., 2014). Here, we discuss the intersection of these two major cellular networks. Specifically, we review the current reported miRNA regulatory interactions on the HSP40 family of chaperones, commonly referred to as J-proteins. Furthermore, we will consider the potential expanded network of interactions predicted 
by bioinformatic analysis. For this study, we will focus on J-proteins which represent the largest and most diverse group of molecular chaperones (Kampinga and Craig, 2010).

\section{MOLECULAR CHAPERONES}

The cellular proteostasis network coordinates protein synthesis, degradation, and stress responses to ensure the correct folding, concentration, and localization of proteins to effectively carry out their cellular functions (Hipp et al., 2014). Molecular chaperones are an integral component of all proteostasis processes. They are a large group of $\sim 300$ proteins (Brehme et al., 2014) that operate to recognize and deal with protein misfolding issues arising throughout the proteome. A multitude of functions is attributed to individual chaperone members with respect to facilitating protein folding, protein disaggregation, sequestration of aggregates, and directing misfolded proteins toward cellular degradation pathways (Kim et al., 2013; Kaushik and Cuervo, 2018; Nillegoda et al., 2018). Individual chaperones can often be grouped into distinct protein families e.g. J-proteins, Hsp60, Hsp70, Hsp90, sHsp. The most well-studied mechanistic aspect of chaperone activities is their ability to reversibly bind, and release unfolded and misfolded substrates (often termed clients) to promote their proper folding and prevent aggregation. Members of the major Heat shock protein 70 (Hsp70) and Heat shock protein 90 (Hsp90) families interact with hundreds of client proteins (Kerner et al., 2005; Taipale et al., 2012). In contrast, some chaperones members of the J-protein family show evidence of more discrete client binding profiles (Fotin et al., 2004; Gong et al., 2009; Kakkar et al., 2016a,b; Craig and Marszalek, 2017). However, chaperone interactions are not limited to client binding. Chaperones are known to work cooperatively with other chaperones and components of the proteostasis network, functioning as larger protein complexes (Taipale et al., 2014; Rizzolo et al., 2017; Freilich et al., 2018; Karunanayake and Page, 2021). For example, HSP70s have ATPase activities that allow for cycling between client binding and release-J-protein and Bag chaperone families strongly stimulate this cycling by promoting ATP hydrolysis and release, respectively (Freilich et al., 2018). Another factor contributing to the complex nature of chaperone activities is the different subcellular localization and expression levels of individual chaperones that directly influence client binding and stoichiometry of formed chaperone complexes (Craig and Marszalek, 2017).

\section{The J-Protein Family of Chaperones. Potential for Regulation to Shape Cellular Proteostasis}

The modular nature of chaperone complexes is proposed to contribute to the fine-tuning of chaperone recruitment and processing of specific clients in the cell. One large family of chaperones that facilitate modularity is the J-protein family. There are over 40 identified J-protein family members in humans (Kampinga and Craig, 2010), which are listed in Table 1. All members share a characteristic J-domain that facilitates modes of Hsp70 binding and ATP hydrolysis (Karzai and McMacken, 1996; Jiang et al., 2007; Kityk et al., 2018; Faust et al., 2020) and thus, are commonly referred to as J-proteins. However, beyond this shared domain, there is incredible diversity between J-protein members with respect to their structural and functional domains: individual J-proteins possess unique combinations of different client binding domains, localization signals, and enzymatic activities (Kampinga and Craig, 2010). J-proteins are roughly classified into three groups by structure. The A-class J-proteins share an overall domain structure similar to the E.coli J-proteins whereas the B-class J-proteins have only partially retained these domains. The remaining J-proteins are simply categorized as C-class (Kampinga and Craig, 2010).

Multiple J-proteins can compete for interactions with the same Hsp70. Therefore, varying the expression levels of individual J-proteins could, in turn, fine-tune the proteostasis of specific client subsets in a cell. Furthermore, there is evidence that changes in the balance of chaperone concentrations can have significant effects on chaperone complex formation and function (Kanelakis et al., 1999; Kundrat and Regan, 2010; Cabrera et al., 2019). For example, increasing the cellular concentrations of the mitochondrial J-protein, DNAJA3, interferes with the ability of the mitochondrial Hsp70 to bind substrates resulting in protein aggregation and mitochondria fragmentation (Lee B. et al., 2015). More broadly, J-proteins may be used to modulate chaperone network function to deal with the specific proteomes of different tissues (Uhlén et al., 2015). Indeed, individual J-proteins do show variations in tissue-specific expression (Hageman and Kampinga, 2009) and mutations in J-proteins are associated with highly tissue-specific diseases (Koutras and Braun, 2014; Sarparanta et al., 2020) such as early-childhood-onset recessive dilated cardiomyopathy and ataxia (Davey et al., 2006; Sparkes et al., 2007) and recessive distal hereditary motor neuropathy (Blumen et al., 2012).

Little is currently known regarding the mechanisms of how cells discretely modulate the expression of J-proteins in a tissuespecific manner or in response to stimuli or stress. Classically studied mechanisms of chaperone regulation are transcription factor activation (e.g., Heat Shock Factor 1) of a broad subset of chaperone gene targets during stress conditions such as heat shock (Zou et al., 1998; Anckar and Sistonen, 2011; Zheng et al., 2016) or ER-stress (Lee et al., 2003; Acosta-Alvear et al., 2007). Nonetheless, while some J-proteins exhibit stressinduced expression, most of the members of the family are constitutively expressed to cell or tissue specific levels (Zhao et al., 2008; Kakkar et al., 2012). In contrast to the stress responseactivated transcription factors, even less is known about the posttranscriptional regulation of chaperone protein expression by other cellular factors such as microRNAs.

Considering the increasing understanding of J-proteins in protein folding-related diseases, such as the reported reduction of several J-proteins in Parkinson's Disease (Hasegawa et al., 2018), a more thorough understanding of the regulation of these proteins is warranted. 
TABLE 1 | J-proteins and their validated miRNA targeting.

\begin{tabular}{|c|c|c|c|c|c|}
\hline Hsp40 & Targeting miRNA & References & Hsp40 & Targeting miRNA & References \\
\hline DNAJA1 & - & - & DNAJC6 & - & - \\
\hline DNAJA2 & - & - & DNAJC7 & - & - \\
\hline DNAJA3 & - & - & DNAJC8 & - & - \\
\hline DNAJA4 & - & - & DNAJC9 & - & - \\
\hline DNAJB1 & miR-370, miR-543 & Evert et al., 2018 & DNAJC10 & - & - \\
\hline DNAJB2a & - & - & DNAJC11 & - & - \\
\hline DNAJB2b & - & - & DNAJC12 & - & - \\
\hline DNAJB4 & - & - & DNAJC13 & - & - \\
\hline DNAJB5 & - & - & DNAJC14 & - & - \\
\hline DNAJB6a & - & - & DNAJC15 & - & - \\
\hline DNAJB6b & - & - & DNAJC16 & - & - \\
\hline DNAJB7 & - & - & DNAJC17 & - & - \\
\hline DNAJB8 & - & - & DNAJC18 & - & - \\
\hline DNAJB9 & miR-25-32-92-363-367 family & Wang et al., 2020 & DNAJC19 & - & - \\
\hline DNAJB11 & miR-29b & Beitzinger et al., 2007 & DNAJC20 & - & - \\
\hline DNAJB12 & miR-148-152 family & Ma et al., 2020 & DNAJC21 & - & - \\
\hline DNAJB13 & - & - & DNAJC22 & - & - \\
\hline DNAJB14 & - & - & DNAJC23 & - & - \\
\hline DNAJC1 & - & - & DNAJC24 & - & - \\
\hline DNAJC2 & - & - & DNAJC25 & - & - \\
\hline DNAJC3 & miR-200 family & Belgardt et al., 2015 & DNAJC26 & - & - \\
\hline DNAJC4 & - & - & DNAJC27 & - & - \\
\hline DNAJC5 & - & - & DNAJC28 & - & - \\
\hline DNAJC5B & - & - & DNAJC29 & - & - \\
\hline DNAJC5G & - & - & DNAJC30 & - & - \\
\hline
\end{tabular}

\section{MicroRNA-MEDIATED SILENCING OF MRNA TRANSCRIPTS}

Since the initial discovery of short non-coding RNAs regulating mRNA translation (Lee et al., 1993; Wightman et al., 1993; Reinhart et al., 2000), it has become apparent that microRNAs (miRNAs) function in the regulation of a large portion of the cellular transcriptome. It is estimated that each miRNA family targets on average more than 400 human mRNAs, and over half of human mRNAs have canonical conserved target sequences in their 3' untranslated regions (UTRs) (Friedman et al., 2009).

Endogenous miRNAs arise from long primary transcripts. A series of cellular processing events, depending on the transcript origin of the miRNA, produce the final mature miRNA in the Ago protein-containing silencing complexes (Bartel, 2018). The mature $\sim 22$ nucleotides (nt) miRNA, guide the Ago proteincontaining complexes to their target mRNAs via base pairing. In canonical targeting of mRNAs, this involves contiguous base pairing of the 5' seed region of the miRNA (nts 2-7) (Bartel, 2018). Base pairing with additional 3' nucleotides in the miRNA can occur but has been reported to have minimal effects on silencing efficacy (Grimson et al., 2007; Wee et al., 2012; Salomon et al., 2015). While several mechanisms have been reported regarding the silencing of mRNAs by miRNAs, the repression mechanism dependent on the TNRC6 adaptor protein family is the dominant mechanism in humans, as recently reviewed and discussed (Jonas and Izaurralde, 2015; Bartel, 2018). In this mechanism, TNRC6 family proteins bind several miRNAago complexes and therefore enhance the silencing of several miRNAs to one mRNA.

\section{CURRENT STATE OF REPORTED MIRNA REGULATORS OF J-PROTEINS}

While there have been numerous reports in the literature describing correlations of J-Protein expression with miRNA expression, there are relatively few examples where the target sequence in the 3' UTR of the J-Protein mRNAs has been experimentally validated (see Table 1). Reports only describing anti-correlations in J-Protein expression with miRNA expression were omitted as indirect regulator interaction networks cannot be ruled out without further investigation. Some of these excluded reports include examples with compelling data where miRNAdependent regulation is through the 3' UTR of a target mRNA, such as the down regulation of DNAJC6 upon miR-146b-5p expression (Kirchmeyer et al., 2018). Cases where the target sequence for a miRNA was not verified were also excluded (Mitra et al., 2012; Yang et al., 2014; Mycko et al., 2015). Increasing complexity of regulation of long non-coding RNAs (lncRNA) (Goodall and Wickramasinghe, 2021) and their interactions with miRNA regulatory networks (López-Urrutia et al., 2019) 
could interfere in the miRNA-mRNA interactions in these cases. We will now briefly summarize verified microRNA targeting of J-proteins.

DNAJB1 is mostly known for the chimeric transcript it forms with PRKACA, which codes for the catalytic domain of protein kinase A in fibrolamellar hepatocellular carcinoma (Honeyman et al., 2014). It furthermore has been argued to be involved in p53-mediated apoptosis through degradation of PDCD5 (Cui et al., 2014). An investigation on a model for Spinocerebellar Ataxia Type 3 (SCA3) revealed a functional role for DNAJB1 in the clearance of mutant polyglutamine (polyQ) protein ataxin-3 aggregates. miR-370 and miR-543, which were both upregulated in SCA3 were shown to specifically target DNAJB1 mRNA. This study highlights possible disease implications miRNAs could have through their interactions with chaperones (Evert et al., 2018).

DNAJC3 is an ER-localized J-protein and co-chaperone to HSPA5. A loss-of-function mutation leads to diabetes mellitus and multisystemic neurodegeneration (Synofzik et al., 2015) and in mice, DNAJC3 knockout mice had a phenotype of partial loss of pancreatic beta-cells (Ladiges et al., 2005). In mouse models of beta cell stress and obesity, miRNA-200 family was found to have a role in promoting the apoptosis of pancreatic beta cells (Belgardt et al., 2015). Transcriptome analysis of miRNA-200 targets in mice revealed DNAJC3 which was then validated as a direct target.

DNAJB9 has recently been shown to inhibit p53-induced apoptosis (Lee H. J. et al., 2015). In models of chemotherapy resistance in acute myeloid leukemia, Wang et al. (2020) identified a regulatory network that involves the direct downregulation of DNAJB9 by miR-32. While miR-32 inhibited DNAJB9, it was in turn modulated by the lncRNA, small nucleolar RNA host gene 5 (SNHG5), creating an axis of control between DNAJB9-miR-32-SNHG5, possibly causing chemotherapy resistance. An analogous regulatory network has also been reported for DNAJB12, an ER-related Jprotein (Ma et al., 2020), where the direct targeting of antiapoptotic DNAJB12 by miR-152-3p is negatively modulated by the lncRNA HCG18 in gastric cancer models. Both studies highlight the complexity of gene regulation through the miRNA network, including the involvement of factors such as lncRNAs.

Additional evidence of miRNA targeting of J-Proteins can be taken where miRNA-Argonaute protein complexes have been identified to associate with the 3' UTRs. As an example, immunoprecipitation experiments revealed DNAJB11 as a component of miR-29-Ago complexes (Beitzinger et al., 2007). While this is strongly suggestive of a regulatory interaction, the authors indicate that not all the interactions identified lead to biological downregulation upon validation.

\section{MicroRNA TARGET PREDICTION OF J-PROTEINS}

While this list of validated regulatory interactions of miRNAs with J-proteins is quite limited, the correlative data in the literature suggests there are significantly more interactions awaiting validation.

To obtain a more global perspective on the potential miRNA network of interactions on the J-Proteins we performed an in silico analysis of miRNA target predictions. To this end, we utilized the most recent version of Targetscan (version 7.2) which identifies predicted canonical mRNA target sequences with 7$8 \mathrm{nt}$ stretches of complementarity to the miRNA seed sequence (Agarwal et al., 2015).

Within the context of miRNA target prediction, both evolutionary conservation aspects of the miRNAs themselves and particular putative miRNA target sequences within a mRNA exist. With respect to our analysis, there are miRNAs and miRNA families that are broadly conserved among vertebrates and miRNAs conserved among mammals (Bartel, 2018). While there may be more recently evolved miRNAs, it has been proposed that many of these have too few targets under selective pressure to enable target predictions with any confidence (Friedman et al., 2009). A major caveat of any predicted miRNA target analyses is that not all mRNAs with 7-8 nt complimentary sites to the miRNA seed sequence exhibit regulation by that miRNA (Baek et al., 2008; Selbach et al., 2008).

An initial analysis for putative miRNA targets of the J-protein family with cumulative context scores of $\leq-0.1$ (Agarwal et al., 2015) as a first-pass threshold, results in 1,337 potential miRNA target sequences for 212 different miRNAs or miRNA families. This minimal criterion yields an unwieldy number of potential sites for experimental validation and most likely consists of a high proportion of false identifications. As mRNAs with evolutionary conserved potential miRNA target sequences in their 3' UTRs exhibit a higher probability of responding to the activity of a miRNA (Baek et al., 2008), strategies to identify more likely miRNA target sequences include choosing sites that exhibit conservation (Bartel, 2009; Friedman et al., 2009). As a result, we applied the criteria for evolutionarily conserved mRNA target sequences for both broadly conserved miRNAs among vertebrates and miRNAs conserved among mammals. This additional parameter reduces the likelihood of false positive identifications in the dataset, while including many possible cross-species interactions. Thus, although our additional analysis criteria decreases our false negative rate of prediction it will also miss some potentially biologically relevant chaperone-miRNA interactions that are not conserved among species. It should be noted that this new criteria also leads to the exclusion of target predictions for some highly probable targets. One example being the targeting of DNAJB5 by miR-21 (Lampis et al., 2018), where miR-21 expression was demonstrated to lead to 3' UTR dependent regulation of DNAJB5. This miR-21:DNAJB5 interaction was excluded from our presentation as a valid target as the predicted target sequence was not experimentally verified. With a selection criterion for conserved putative mRNA target sites for conserved miRNAs, Targetscan identifies 164 and 72 predicted targets respectively for either broadly conserved miRNAs or miRNAs conserved among mammals. This level of analysis reveals significant variations between the members of the J-protein family. As seen in Figure 1, nine mRNAs have no predicted canonical miRNA target sequences, such as DNAJA3, 


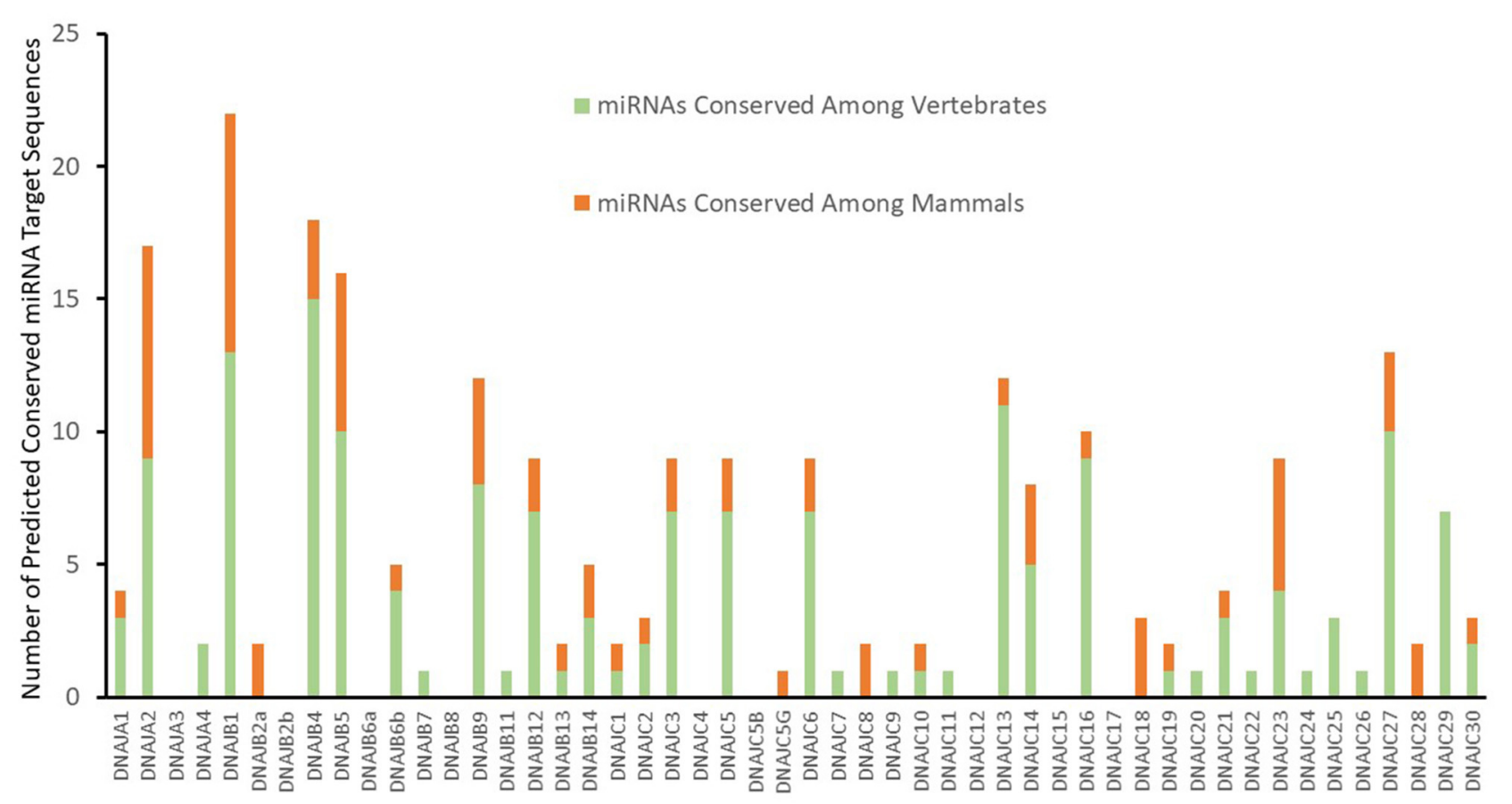

FIGURE 1 | Predicted targeting by conserved miRNA Families. Predicted conserved J-Protein target sites of miRNAs from TargetScan (Agarwal et al., 2015) with a context score of $<-0.1$. The number of predicted conserved miRNA targets for each of the J-proteins are indicated. The proportion of predicted targets by miRNAs and miRNA families broadly conserved throughout vertebrates or only within mammals are also indicated.

DNAJB8, and DNAJC4. On the opposite side of the spectrum, several J-Protein mRNAs contain a high number of predicted conserved miRNA targets. The mRNAs for DNAJA2, DNAJB1, DNAJB4, and DNAJB5 each have $>15$ predicted conserved miRNA target sequences within their 3' UTRs. Among these, only two of the predicted target sequences for DNAJB1 have been experimentally verified (Evert et al., 2018).

The specific predicted conserved target interactions for all the J-Protein members by broadly conserved miRNAs or those conserved among mammals are shown in Figures 2, 3 respectively. With the reported known interactions listed in Table 1 highlighted in these figures, it is apparent how few of these predicted 234 interactions have been investigated. This therefore emphasizes an area of research in chaperone biology that is primed for further investigation.

\section{Predicting Strong miRNA Candidates of J-Protein Regulation}

Despite the large number of predicted potential interactions, many predicted conserved miRNA target sequences are known to not significantly regulate expression levels of their targets in cells (Baek et al., 2008). Proteomic analysis of the same miRNAs in different cellular backgrounds reveals cell-specific differences (Ludwig et al., 2016; Piragasam et al., 2020) and changes in protein abundances that may counter interactions shown by miRNA target predictions (Piragasam et al., 2020). These proteomic analyses are holistic in that they reveal both direct and indirect impacts on protein abundance through
miRNAs. Results that counter predictions can thus not be used to rule out potential interactions. Nonetheless, these types of analyses may provide some insight and guidance for predicting interactions that might lead to stronger regulation of a given target which can then be prioritized for investigation. In the next few paragraphs, we examine two potential miRNA mechanisms that may enhance their effects on target transcripts and highlight potential examples in our J-protein-miRNA dataset.

\section{Multiple miRNA Target Sites}

The degree of regulation of mRNAs by miRNAs depends on the identities and abundance of the particular miRNAs in the cell, the number of target mRNA sites in a cell, as well as the specific binding efficacy for a given miRNA target site. For each given miRNA:mRNA interaction, the regulation is typically modest with repression being $<50 \%$ (Baek et al., 2008; Selbach et al., 2008). However, enhanced repression is often observed when multiple miRNA target sites are within the same 3' UTR as these effects are typically additive. The predictions presented in Figures 2, 3 reveal several individual miRNAs that are predicted to have multiple mRNA target sequences within a given 3' UTR. These include the three predicted target sites for miR23-3p within the 3' UTR of DNAJC6 and an additional 12 miRNA:mRNA interactions with two predicted miRNA target sites. Of the 12 predictions with two predicted target sites in an mRNA for the same miRNA, the targeting of DNAJB1 by miR370 (Evert et al., 2018) and DNAJC3 by the miRNA-200 family (Belgardt et al., 2015) have been validated. 


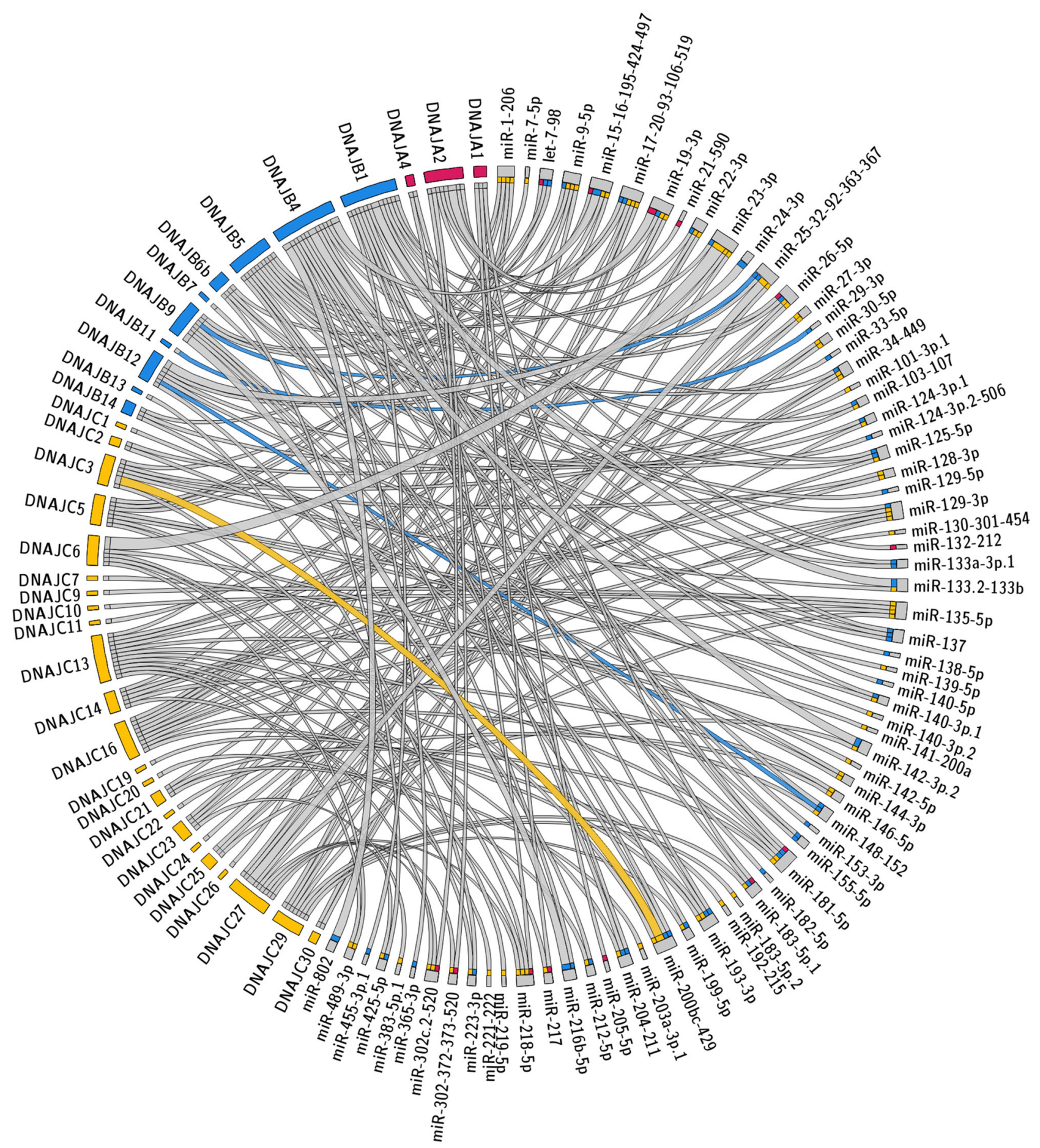

FIGURE 2 | Predicted miRNA targets for miRNAs broadly conserved in vertebrates. Interaction Circos plot (Krzywinski et al., 2009) for TargetScan depicting targeting of J-Proteins by miRNA families that are broadly conserved throughout vertebrates. For each J-Protein, miRNAs with a context score of $<-0.1$ were extracted from TargerScan (Agarwal et al., 2015). Unconserved target sequences for miRNAs that were not conserved were excluded from the analysis. A colorized line indicates miRNA targeting that has been experimentally verified and the width of the bridging lines is proportional to the number of predicted target sequences.

\section{Cooperative miRNA Targeting}

Complex patterns of miRNA expression exist in different cell and tissue types (Landgraf et al., 2007; Chaulk et al., 2016). The intersection of both miRNA and mRNA expression patterns can yield increasingly complex combinatorial regulatory networks of regulation. This regulation becomes even more complex when considering that multiple miRNAs can simultaneously target the same 3' UTR of an mRNA and lead to differential outcomes 


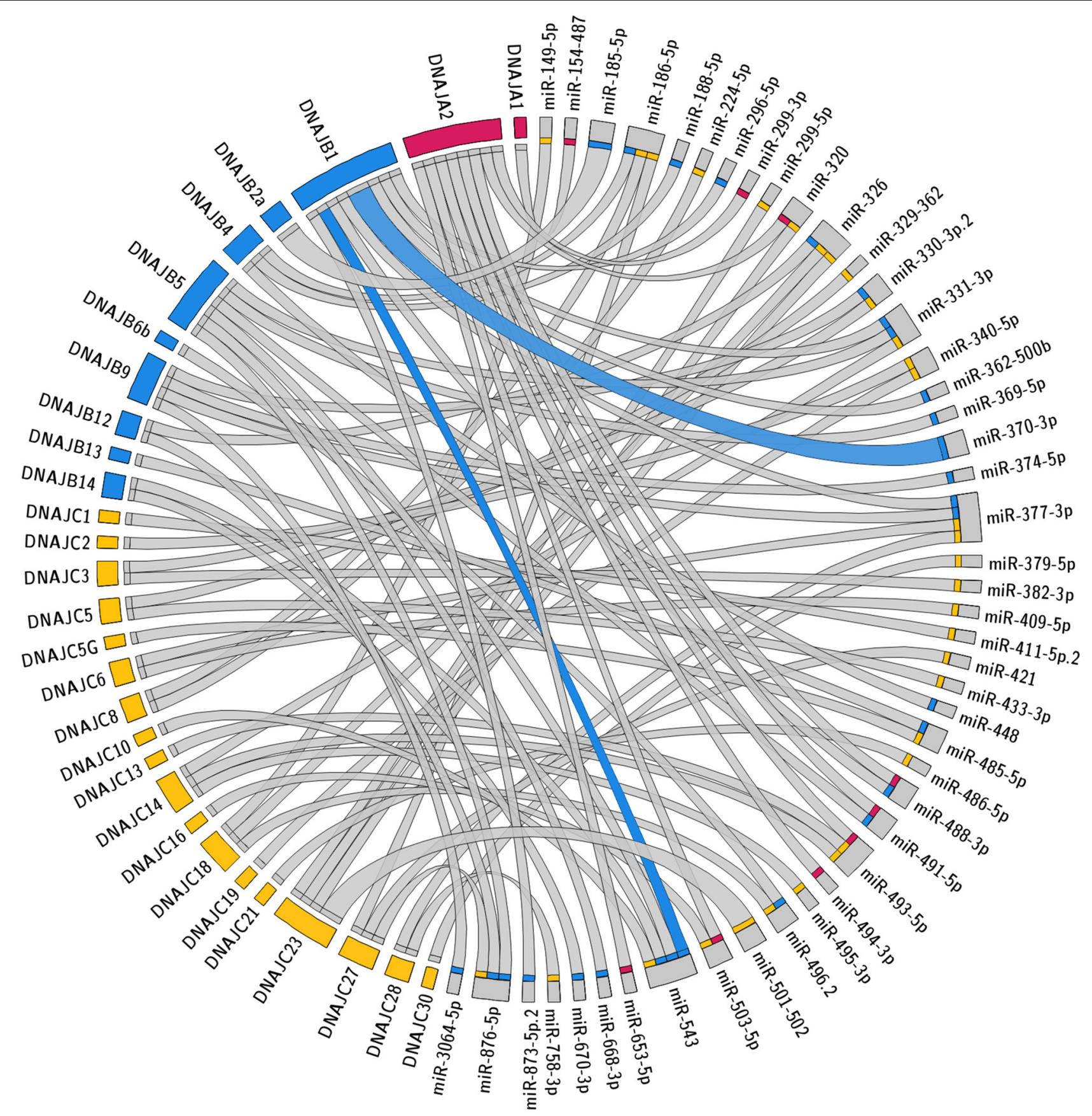

FIGURE 3 | Predicted miRNA targets for miRNAs conserved within mammals. Interaction Circos plot (Krzywinski et al., 2009) for TargetScan depicting miRNA targeting of the J-Protein by miRNA families that are conserved throughout mammals. For each J-Protein, miRNAs with a context score of $<-0.1$ were extracted from TargerScan (Agarwal et al., 2015). Unconserved target sequences for miRNAs that were not conserved were excluded from the analysis. A colorized line indicates miRNA targeting that has been experimentally verified and the width of the bridging lines is proportional to the number of predicted target sequences.

for the same transcript depending on a cell's given miRNA signature pattern.

The potential for multiple different miRNAs targeting the same J-Protein is summarized in Figures 2, 3. Here it should be noted that the co-targeting of DNAJB1 by miR-543 and miR370 has been documented (Evert et al., 2018). There is another level of complexity regarding co-targeting that is not revealed in the presentation of the figures, that is, their spatial proximity. In some cases, miRNAs are reported to act cooperatively on the same mRNA, specifically those with target sequences within 8$40 \mathrm{nt}$ of each other (Grimson et al., 2007; Sætrom et al., 2007). This is a result of the TNRC6 proteins being able to associate with 


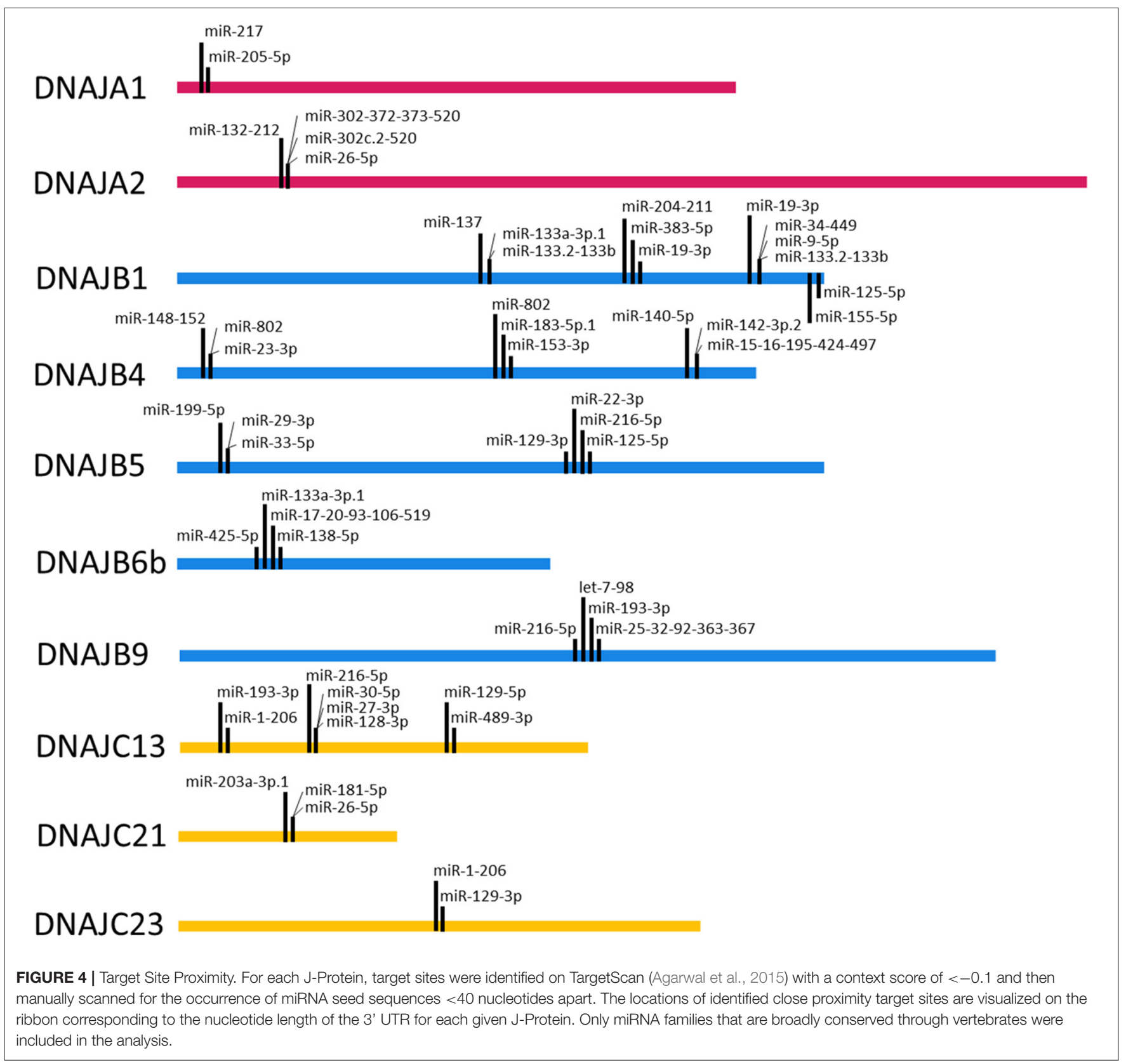

multiple Ago protein complexes simultaneously (Briskin et al., 2020).

To query the potential for cooperative interactions between the predicted miRNA target sequences in the 3' UTRs of the J-protein mRNAs, target sequences within 8-40 nts of each other were identified. Figure 4 depicts the miRNA target sequences that meet this criterion for the miRNA families broadly conserved among vertebrates. Intriguingly, the analysis reveals a potential for another level of complexity with regard to miRNA regulation. While the analysis identified multiple examples of potential miRNA target sequences within 8-40 nts, such as the predicted miR-217 and miR-205-5p target sequences in the 3' UTR of the DNAJA1 mRNA, there are also potential combinations of mutual exclusivity. For example, as shown in Figure 4, the DNAJB4 mRNA has predicted miRNA target sequences for the miR-148-152 family within 40 nts of the predicted target sequences for miR-802 and miR-23-3p. As the predicted target sequences for miR-802 and miR-23-3p partially overlap, then if both sequences exhibit bonafide mRNA targeting in cells, they would have to be mutually exclusive in their targeting by these miRNAs. This leads to the prediction that miR-148-152 could act cooperatively with either miR-802 and miR-23-3p but that these two miRNAs could not bind to the same DNAJB4 mRNA to regulate its expression. While this form of potential regulation leads to numerous instances of a Boolean logic type of regulation behavior, it is currently unclear whether this behavior would be 
recapitulated in cells and would be highly dependent on miRNA complex concentrations.

\section{CONCLUSION}

Perhaps as a result of the enormous inherent complexities of both the chaperone networks and miRNA regulatory networks, there has been relatively little reported work validating their intersection of regulation. Our analysis offers starting points for the exploration of miRNA and J-protein interactions. With the reported linkages and interest in both miRNAs and J-proteins in human diseases such as neurodegeneration and cancer, there are ample possibilities that we have outlined for future investigations into the interplay of these systems.

\section{REFERENCES}

Acosta-Alvear, D., Zhou, Y., Blais, A., Tsikitis, M., Lents, N. H., Arias, C., et al. (2007). XBP1 controls diverse cell type- and condition-specific transcriptional regulatory networks. Mol. Cell 27, 53-66. doi: 10.1016/j.molcel.2007.06.011

Agarwal, V., Bell, G. W., Nam, J., and Bartel, D. P. (2015). Predicting effective microRNA target sites in mammalian mRNAs. eLife 4:e05005. doi: 10.7554/eLife.05005

Anckar, J., and Sistonen, L. (2011). Regulation of HSF1 function in the heat stress response: implications in aging and disease. Annu. Rev. Biochem. 80, 1089-1115. doi: 10.1146/annurev-biochem-060809-095203

Baek, D., Shin, C., Gygi, S. P., Camargo, F. D., Villien, J., and Bartel, D. P. (2008). The impact of microRNAs on protein output. Nature 455, 64-71. doi: 10.1038 /nature 07242

Bartel, D. P. (2009). MicroRNAs: target recognition and regulatory functions. Cell 136, 215-233. doi: 10.1016/j.cell.2009.01.002

Bartel, D. P. (2018). Metazoan microRNAs. Cell 173, 20-51. doi: 10.1016/j.cell.2018.03.006

Beitzinger, M., Peters, L., Zhu, J. Y., Kremmer, E., and Meister, G. (2007). Identification of human microRNA targets from isolated argonaute protein complexes. RNA Biol. 4, 76-84. doi: 10.4161/rna.4.2.4640

Belgardt, B., Ahmed, K., Spranger, M., Latreille, M., Denzler, R., Kondratiuk, N., et al. (2015). The microRNA-200 family regulates pancreatic beta cell survival in type 2 diabetes. Nat. Med. 21, 619-627. doi: 10.1038/nm.3862

Blumen, S. C., Astord, S., Robin, V., Vignaud, L., Toumi, N., Cieslik, A., et al. (2012). A rare recessive distal hereditary motor neuropathy with HSJ1 chaperone mutation. Ann. Neurol. 71, 509-519. doi: 10.1002/ana.22684

Brehme, M., Voisine, C., Rolland, T., Wachi, S., Soper, J., Zhu, Y., et al. (2014). A chaperome subnetwork safeguards proteostasis in aging and neurodegenerative disease. Cell Rep. 9, 1135-1150. doi: 10.1016/j.celrep. 2014.09.042

Briskin, D., Wang, P. Y., and Bartel, D. P. (2020). The biochemical basis for the cooperative action of microRNAs. Proc. Natl. Acad. Sci. U.S.A. 117, 17764-17774. doi: 10.1073/pnas.1920404117

Cabrera, Y., Dublang, L., Fernández-Higuero, J. A., Albesa-Jové, D., Lucas, M., Viguera, A. R., et al. (2019). Regulation of human Hsc70 ATPase and chaperone activities by Apg2: role of the acidic subdomain. J. Mol. Biol. 431, 444-461. doi: $10.1016 /$ j.jmb.2018.11.026

Chaulk, S. G., Ebhardt, H. A., and Fahlman, R. P. (2016). Correlations of microRNA:microRNA expression patterns reveal insights into microRNA clusters and global microRNA expression patterns. Mol. BioSyst. 12, 110-119. doi: $10.1039 / \mathrm{C} 5 \mathrm{MB} 00415 \mathrm{~B}$

Craig, E. A., and Marszalek, J. (2017). How do J-proteins get Hsp70 to do so many different things? Trends Biochem. Sci. 42, 355-368. doi: 10.1016/j.tibs.2017.02.007

Cui, X., Choi, H., Choi, Y., Park, S., Sung, G., Lee, Y., et al. (2014). DNAJB1 destabilizes PDCD5 to suppress p53-mediated apoptosis. Cancer Lett. 357, 307-315. doi: 10.1016/j.canlet.2014.11.041

\section{AUTHOR CONTRIBUTIONS}

LB: drafted the manuscript and collected literature and data. RF: data analysis and edited the manuscript. SAM: designed the content of the article and edited the manuscript. All authors contributed to the article and approved the submitted version.

\section{FUNDING}

This project was supported by NSERC Discovery Grant program funding to SAM. LB was supported by fellowships from the Neuroscience and Mental Health Institute (University of Alberta) and SynAD (University of Alberta).

Davey, K. M., Parboosingh, J. S., McLeod, D. R., Chan, A., Casey, R., Ferreira, P., et al. (2006). Mutation of DNAJC19, a human homologue of yeast inner mitochondrial membrane co-chaperones, causes DCMA syndrome, a novel autosomal recessive Barth syndrome-like condition. J. Med. Genet. 43, 385-393. doi: 10.1136/jmg.2005.036657

Evert, B. O., Nalavade, R., Jungverdorben, J., Matthes, F., Weber, S., Rajput, A., et al. (2018). Upregulation of miR-370 and miR-543 is associated with reduced expression of heat shock protein 40 in spinocerebellar ataxia type 3. PLOS ONE 13:e201794. doi: 10.1371/journal.pone.0201794

Faust, O., Abayev-Avraham, M., Wentink, A. S., Maurer, M., Nillegoda, N. B., London, N., et al. (2020). HSP40 proteins use class-specific regulation to drive HSP70 functional diversity. Nature 587, 489-494. doi: 10.1038/s41586-020-2906-4

Fotin, A., Cheng, Y., Grigorieff, N., Walz, T., Harrison, S. C., and Kirchhausen, T. (2004). Structure of an auxilin-bound clathrin coat and its implications for the mechanism of uncoating. Nature 432, 649-653. doi: 10.1038/nature03078

Freilich, R., Arhar, T., Abrams, J. L., and Gestwicki, J. E. (2018). Protein-protein interactions in the molecular chaperone network. Acc. Chem. Res. 51, 940-949. doi: 10.1021/acs.accounts.8b00036

Friedman, R. C., Farh, K. K., Burge, C. B., and Bartel, D. P. (2009). Most mammalian mRNAs are conserved targets of microRNAs. Genome Res. 19, 92-105. doi: 10.1101/gr.082701.108

Gong, Y., Zhang, Z., Kakihara, Y., Emili, A., Krogan, N., Houry, W. A., et al. (2009). An atlas of chaperone-protein interactions in Saccharomyces cerevisiae: implications to protein folding pathways in the cell. Mol. Syst. Biol. 5:275. doi: $10.1038 / \mathrm{msb} .2009 .26$

Goodall, G. J., and Wickramasinghe, V. O. (2021). RNA in cancer. Nat. Rev. Cancer 21, 22-36. doi: 10.1038/s41568-020-00306-0

Grimson, A., Farh, K. K., Johnston, W. K., Garrett-Engele, P., Lim, L. P., and Bartel, D. P. (2007). MicroRNA targeting specificity in mammals: determinants beyond seed pairing. Mol. Cell 27, 91-105. doi: 10.1016/j.molcel.2007.06.017

Hageman, J., and Kampinga, H. H. (2009). Computational analysis of the human HSPH/HSPA/DNAJ family and cloning of a human HSPH/HSPA/DNAJ expression library. Cell Stress Chaperones 14, 1-21. doi: 10.1007/s12192-008-0060-2

Hasegawa, T., Yoshida, S., Sugeno, N., Kobayashi, J., and Aoki, M. (2018). DnaJ/Hsp40 family and parkinson's disease. Front. Neurosci. 11:743. doi: $10.3389 /$ fnins.2017.00743

Hipp, M. S., Park, S., and Hartl, F. U. (2014). Proteostasis impairment in protein-misfolding and -aggregation diseases. Trends Cell Biol. 24, 506-514. doi: $10.1016 /$ j.tcb.2014.05.003

Honeyman, J. N., Simon, E. P., Robine, N., Chiaroni-Clarke, R., Darcy, D. G., Lim, I. I. P., et al. (2014). Detection of a recurrent DNAJB1-PRKACA chimeric transcript in fibrolamellar hepatocellular carcinoma. Science 343, 1010-1014. doi: $10.1126 /$ science. 1249484

Jiang, J., Maes, E. G., Taylor, A. B., Wang, L., Hinck, A. P., Lafer, E. M., et al. (2007). Structural basis of J cochaperone binding and regulation of Hsp70. Mol. Cell 28, 422-433. doi: 10.1016/j.molcel.2007.08.022 
Jonas, S., and Izaurralde, E. (2015). Towards a molecular understanding of microRNA-mediated gene silencing. Nat. Rev. Genet. 16, 421-433. doi: $10.1038 / \mathrm{nrg} 3965$

Kakkar, V., Kuiper, E. F. E., Pandey, A., Braakman, I., and Kampinga, H. H. (2016a). Versatile members of the DNAJ family show Hsp70 dependent antiaggregation activity on RING1 mutant parkin C289G. Sci. Rep. 6:34830. doi: $10.1038 /$ srep 34830

Kakkar, V., Månsson, C., de Mattos, E., Bergink, S., van der Zwaag, M., van Waarde, M. W. H., et al. (2016b). The S/T-rich motif in the DNAJB6 chaperone delays polyglutamine aggregation and the onset of disease in a mouse model. Mol. Cell 62, 272-283. doi: 10.1016/j.molcel.2016.03.017

Kakkar, V., Prins, L. C. B., and Kampinga, H. H. (2012). DNAJ Proteins and protein aggregation diseases. Curr. Top. Med. Chem. 12, 2479-2490. doi: $10.2174 / 1568026611212220004$

Kampinga, H. H., and Craig, E. A. (2010). The HSP70 chaperone machinery: J proteins as drivers of functional specificity. Nat. Rev. Mol. Cell Biol. 11, 579-592. doi: $10.1038 / \mathrm{nrm} 2941$

Kanelakis, K. C., Morishima, Y., Dittmar, K. D., Galigniana, M. D., Takayama, S., Reed, J. C., et al. (1999). Differential effects of the hsp70-binding protein BAG-1 on glucocorticoid receptor folding by the hsp90-based chaperone machinery. $J$. Biol. Chem. 274, 34134-34140. doi: 10.1074/jbc.274.48.34134

Karunanayake, C., and Page, R. C. (2021). Cytosolic protein quality control machinery: Interactions of Hsp70 with a network of co-chaperones and substrates. Exp. Biol. Med. doi: 10.1177/1535370221999812. [Epub ahead of print].

Karzai, A. W., and McMacken, R. (1996). A bipartite signaling mechanism involved in dnaj-mediated activation of the Escherichia coli DnaK protein. J. Biol. Chem. 271, 11236-11246. doi: 10.1074/jbc.271.19.11236

Kaushik, S., and Cuervo, A. M. (2018). The coming of age of chaperone-mediated autophagy. Nat. Rev. Mol. Cell Biol. 19, 365-381. doi: 10.1038/s41580-018-0001-6

Kerner, M. J., Naylor, D. J., Ishihama, Y., Maier, T., Chang, H., Stines, A. P., et al. (2005). Proteome-wide analysis of chaperonin-dependent protein folding in Escherichia coli. Cell 122, 209-220. doi: 10.1016/j.cell.2005.05.028

Kim, Y. E., Hipp, M. S., Bracher, A., Hayer-Hartl, M., and Hartl, F. U. (2013). Molecular chaperone functions in protein folding and proteostasis. Ann. Rev. Biochem. 82, 323-355. doi: 10.1146/annurev-biochem-060208-0 92442

Kirchmeyer, M., Servais, F. A., Hamdorf, M., Nazarov, P. V., Ginolhac, A., Halder, R., et al. (2018). Cytokine-mediated modulation of the hepatic miRNome: miR146b-5p is an IL-6-inducible miRNA with multiple targets. J. Leukoc. Biol. 104, 987-1002. doi: 10.1002/JLB.MA1217-499RR

Kityk, R., Kopp, J., and Mayer, M. P. (2018). Molecular mechanism of J-domaintriggered ATP hydrolysis by Hsp70 chaperones. Mol. Cell 69, 227-237.e4. doi: 10.1016/j.molcel.2017.12.003

Koutras, C., and Braun, J. E. A. (2014). J protein mutations and resulting proteostasis collapse. Front. Cell. Neurosci. 8:191. doi: 10.3389/fncel.2014.00191

Krzywinski, M., Schein, J., Birol, I., Connors, J., Gascoyne, R., Horsman, D., et al. (2009). Circos: an information aesthetic for comparative genomics. Genome Res. 19, 1639-1645. doi: 10.1101/gr.092759.109

Kundrat, L., and Regan, L. (2010). Balance between folding and degradation for Hsp90-dependent client proteins: a key role for CHIP. Biochemistry 49, 7428-7438. doi: 10.1021/bi100386w

Ladiges, W. C., Knoblaugh, S. E., Morton, J. F., Korth, M. J., Sopher, B. L., Baskin, C. R., et al. (2005). Pancreatic $\beta$-cell failure and diabetes in mice with a deletion mutation of the endoplasmic reticulum molecular chaperone gene P58IPK. Diabetes 54, 1074-1081. doi: 10.2337/diabetes.54.4.1074

Lampis, A., Carotenuto, P., Vlachogiannis, G., Cascione, L., Hedayat, S., Burke, R., et al. (2018). MIR21 drives resistance to heat shock protein 90 inhibition in cholangiocarcinoma. Gastroenterology 154, 1066-1079.e5. doi: 10.1053/j.gastro.2017.10.043

Landgraf, P., Rusu, M., Sheridan, R., Sewer, A., Iovino, N., Aravin, A., et al. (2007). A mammalian microRNA expression atlas based on small RNA library sequencing. Cell 129, 1401-1414. doi: 10.1016/j.cell.2007.04.040

Lee, A., Iwakoshi, N. N., and Glimcher, L. H. (2003). XBP-1 regulates a subset of endoplasmic reticulum resident chaperone genes in the unfolded protein response. Mol. Cell. Biol. 23, 7448-7459. doi: 10.1128/MCB.23.21.7448-7459.2003
Lee, B., Ahn, Y., Kang, S., Park, Y., Jeon, Y., Rho, J. M., et al. (2015). Stoichiometric expression of mtHsp40 and mtHsp70 modulates mitochondrial morphology and cristae structure via Opa1L cleavage. Mol. Biol. Cell 26, 2156-2167. doi: 10.1091/mbc.E14-02-0762

Lee, H. J., Kim, J. M., Kim, K. H., Heo, J. I., Kwak, S. J., and Han, J. A. (2015). Genotoxic stress/p53-induced DNAJB9 inhibits the pro-apoptotic function of p53. Cell Death Differ. 22, 86-95. doi: 10.1038/cdd.2014.116

Lee, R. C., Feinbaum, R. L., and Ambros, V. (1993). The C. elegans heterochronic gene lin-4 encodes small RNAs with antisense complementarity to lin-14. Cell 75, 843-854. doi: 10.1016/0092-8674(93)90529-Y

López-Urrutia, E., Bustamante Montes, L. P., Ladrón de Guevara Cervantes, D., Pérez-Plasencia, C., and Campos-Parra, A. D. (2019). Crosstalk between long non-coding RNAs, micro-RNAs and mRNAs: deciphering molecular mechanisms of master regulators in cancer. Front. Oncol. 9:669. doi: 10.3389/fonc.2019.00669

Ludwig, K. R., Dahl, R., and Hummon, A. B. (2016). Evaluation of the mirn23a cluster through an iTRAQ-based quantitative proteomic approach. J. Proteome Res. 15, 1497-1505. doi: 10.1021/acs.jproteome.5b01101

Ma, P., Li, L., Liu, F., and Zhao, Q. (2020). HNF1A-induced lncRNA HCG18 facilitates gastric cancer progression by upregulating DNAJB12 via miR-152-3p. OncoTargets Ther. 13, 7641-7652. doi: 10.2147/OTT.S253391

Mitra, A., Rostas, J. W., Dyess, D. L., Shevde, L. A., and Samant, R. S. (2012). MicroRNA-632 downregulates DNAJB6 in breast cancer. Lab. Invest. 92, 1310-1317. doi: 10.1038/labinvest.2012.87

Mycko, M. P., Cichalewska, M., Cwiklinska, H., and Selmaj, K. W. (2015). miR-155-3p drives the development of autoimmune demyelination by regulation of heat shock protein 40. J. Neurosci. 35, 16504-16515. doi: 10.1523/JNEUROSCI.2830-15.2015

Nillegoda, N. B., Wentink, A. S., and Bukau, B. (2018). Protein disaggregation in multicellular organisms. Trends Biochem. Sci. 43, 285-300. doi: 10.1016/j.tibs.2018.02.003

Piragasam, R. S., Hussain, S. F., Chaulk, S. G., Siddiqi, Z. A., and Fahlman, R. P. (2020). Label-free proteomic analysis reveals large dynamic changes to the cellular proteome upon expression of the miRNA-23a-27a-24-2 microRNA cluster. Biochem. Cell Biol. 98, 61-69. doi: 10.1139/bcb-2019-0014

Reinhart, B. J., Pasquinelli, A. E., Bettinger, J. C., Horvitz, H. R., Basson, M., Ruvkun, G., et al. (2000). The 21-nucleotide let-7 RNA regulates developmental timing in Caenorhabditis elegans. Nature 403, 901-906. doi: 10.1038/35002607

Rizzolo, K., Huen, J., Kumar, A., Phanse, S., Vlasblom, J., Kakihara, Y., et al. (2017). Features of the chaperone cellular network revealed through systematic interaction mapping. Cell Rep. 20, 2735-2748. doi: 10.1016/j.celrep.2017.08.074

Sætrom, P., Heale, B. S. E., Snøve, O., Aagaard, L., Alluin, J., and Rossi, J. J. (2007). Distance constraints between microRNA target sites dictate efficacy and cooperativity. Nucleic Acids Res. 35, 2333-2342. doi: 10.1093/nar/gkm133

Salomon, W., Jolly, S., Moore, M., Zamore, P., and Serebrov, V. (2015). Singlemolecule imaging reveals that argonaute reshapes the binding properties of its nucleic acid guides. Cell 162, 84-95. doi: 10.1016/j.cell.2015.06.029

Sarparanta, J., Jonson, P. H., Kawan, S., and Udd, B. (2020). Neuromuscular diseases due to chaperone mutations: a review and some new results. Int. J. Mol. Sci. 21:1409. doi: 10.3390/ijms21041409

Selbach, M., Fang, Z., Schwanhäusser, B., Khanin, R., Thierfelder, N., and Rajewsky, N. (2008). Widespread changes in protein synthesis induced by microRNAs. Nature 455, 58-63. doi: 10.1038/nature07228

Sparkes, R., Patton, D., and Bernier, F. (2007). Cardiac features of a novel autosomal recessive dilated cardiomyopathic syndrome due to defective importation of mitochondrial protein. Cardiol. Young 17, 215-217. doi: 10.1017/S1047951107000042

Synofzik, M., Haack, T., Kopajtich, R., Gorza, M., Rapaport, D., Greiner, M., et al. (2015). Absence of BiP co-chaperone DNAJC3 causes diabetes mellitus and multisystemic neurodegeneration. Am. J. Hum. Genet. 96:514. doi: 10.1016/j.ajhg.2015.02.003

Taipale, M., Krykbaeva, I., Koeva, M., Kayatekin, C., Westover, K., Karras, G., et al. (2012). Quantitative analysis of Hsp90-client interactions reveals principles of substrate recognition. Cell 150, 987-1001. doi: 10.1016/j.cell.2012.06.047

Taipale, M., Tucker, G., Peng, J., Krykbaeva, I., Lin, Z., Larsen, B., et al. (2014). A quantitative chaperone interaction network reveals the architecture of cellular protein homeostasis pathways. Cell 158, 434-448. doi: $10.1016 /$ j.cell.2014.05.039 
Uhlén, M., Fagerberg, L., Hallström, B. M., Lindskog, C., Oksvold, P., Mardinoglu, A., et al. (2015). Proteomics. Tissue-based map of the human proteome. Science 347:1260419. doi: 10.1126/science.1260419

Wang, D., Zeng, T., Lin, Z., Yan, L., Wang, F., Tang, L., et al. (2020). Long non-coding RNA SNHG5 regulates chemotherapy resistance through the miR-32/DNAJB9 axis in acute myeloid leukemia. Biomed. Pharmacother. 123:109802. doi: 10.1016/j.biopha.2019.109802

Wee, L., Flores-Jasso, C., Salomon, W., and Zamore, P. (2012). Argonaute divides its RNA guide into domains with distinct functions and RNA-binding properties. Cell 151, 1055-1067. doi: 10.1016/j.cell.2012.10.036

Wightman, B., Ha, I., and Ruvkun, G. (1993). Posttranscriptional regulation of the heterochronic gene lin-14 by lin- 4 mediates temporal pattern formation in C. elegans. Cell 75, 855-862. doi: 10.1016/0092-8674(93) 90530-4

Yang, Z., He, M., Wang, K., Sun, G., Tang, L., and Xu, Z. (2014). Tumor suppressive microRNA-193b promotes breast cancer progression via targeting DNAJC13 and RAB22A. Int. J. Clin. Exp. Pathol. 7, 7563-7570.

Zhao, X., Braun, A., and Braun, J. (2008). Biological roles of neural J proteins. Cell Mol. Life Sci. 65, 2385-2396. doi: 10.1007/s00018-008-8089-z
Zheng, X., Krakowiak, J., Patel, N., Beyzavi, A., Ezike, J., Khalil, A. S., et al. (2016). Dynamic control of Hsfl during heat shock by a chaperone switch and phosphorylation. eLife 5:e18638. doi: 10.7554/eLife.18638

Zou, J., Guo, Y., Guettouche, T., Smith, D. F., and Voellmy, R. (1998). Repression of heat shock transcription factor HSF1 activation by HSP90 (HSP90 complex) that forms a stress-sensitive complex with HSF1. Cell 94, 471-480. doi: 10.1016/S0092-8674(00)81588-3

Conflict of Interest: The authors declare that the research was conducted in the absence of any commercial or financial relationships that could be construed as a potential conflict of interest.

Copyright (c) 2021 Budrass, Fahlman and Mok. This is an open-access article distributed under the terms of the Creative Commons Attribution License (CC BY). The use, distribution or reproduction in other forums is permitted, provided the original author(s) and the copyright owner(s) are credited and that the original publication in this journal is cited, in accordance with accepted academic practice. No use, distribution or reproduction is permitted which does not comply with these terms. 